\title{
Chemically Aware Model Builder (camb): an R package for property and bioactivity modelling of small molecules
}

Daniel S Murrell ${ }^{1 \dagger}$, Isidro Cortes-Ciriano ${ }^{2 \dagger}$, Gerard J P van Westen ${ }^{3}$, Ian P Stott ${ }^{4}$, Andreas Bender ${ }^{1}$ Thérèse E Malliavin ${ }^{2 *}$ and Robert C Glen ${ }^{1 *}$

\begin{abstract}
Background: In silico predictive models have proved to be valuable for the optimisation of compound potency, selectivity and safety profiles in the drug discovery process.

Results: camb is an R package that provides an environment for the rapid generation of quantitative StructureProperty and Structure-Activity models for small molecules (including QSAR, QSPR, QSAM, PCM) and is aimed at both advanced and beginner $\mathrm{R}$ users. camb's capabilities include the standardisation of chemical structure representation, computation of 905 one-dimensional and 14 fingerprint type descriptors for small molecules, 8 types of amino acid descriptors, 13 whole protein sequence descriptors, filtering methods for feature selection, generation of predictive models (using an interface to the R package caret), as well as techniques to create model ensembles using techniques from the R package caretEnsemble). Results can be visualised through high-quality, customisable plots (R package ggplot2).

Conclusions: Overall, camb constitutes an open-source framework to perform the following steps: (1) compound standardisation, (2) molecular and protein descriptor calculation, (3) descriptor pre-processing and model training, visualisation and validation, and (4) bioactivity/property prediction for new molecules. camb aims to speed model generation, in order to provide reproducibility and tests of robustness. QSPR and proteochemometric case studies are included which demonstrate camb's application.
\end{abstract}

Keywords: R, Package, Ensemble, Learning, Workflow, QSPR, QSAR, PCM

\section{Background}

The advent of high-throughput technologies over the last two decades has led to a vast increase in the number of compound and bioactivity databases [1-3]. This increase in the amount of chemical and biological information has been exploited by developing fields in drug discovery such as quantitative structure activity relationships (QSAR), quantitative structure property relationships

\footnotetext{
*Correspondence: therese.malliavin@pasteur.fr; rcg28@cam.ac.uk ${ }^{\dagger}$ Daniel S Murrell and Isidro Cortes-Ciriano contributed equally to this work

${ }^{1}$ Department of Chemistry, Centre for Molecular Informatics, University of Cambridge, Lensfield Road, Cambridge CB2 1EW, UK

${ }^{2}$ Unite de Bioinformatique Structurale, Structural Biology and Chemistry Department, Institut Pasteur and CNRS UMR 3825, 25-28, rue Dr. Roux, 75724 Paris, France

Full list of author information is available at the end of the article
}

(QSPR), quantitative sequence-activity modelling (QSAM), or proteochemometric modelling (PCM) $[4,5]$.

The $R$ programming environment provides a flexible and open platform for statistical analyses [6]. $\mathrm{R}$ is extensively used in genomics [7], and the availability of $R$ packages for cheminformatics and medicinal chemistry is small in comparison. Nonetheless, R currently constitutes the most frequent choice in the medicinal chemistry literature for compound bioactivity and property modelling [8]. In general, these studies share a common algorithmic structure, which can be summarised in four model generation steps: (1) compound standardisation, (2) descriptor calculation, (3) pre-processing, feature selection, model training and validation, and (4) bioactivity/property prediction for new molecules. Fig. 1 illustrates these steps.

\section{Chemistry Central}

(C) 2015 Murrell et al. This article is distributed under the terms of the Creative Commons Attribution 4.0 International License (http://creativecommons.org/licenses/by/4.0/), which permits unrestricted use, distribution, and reproduction in any medium, provided you give appropriate credit to the original author(s) and the source, provide a link to the Creative Commons license, and indicate if changes were made. The Creative Commons Public Domain Dedication waiver (http://creativecommons.org/ publicdomain/zero/1.0/) applies to the data made available in this article, unless otherwise stated. 


Input data
Compounds
Big. $\mathbf{1}$ Overview of camb functionalities. camb provides an open and seamless framework for bioactivity/property modelling (QSAR, QSPR, QSAM
and PCM) including: (1) compound standardisation, (2) molecular and protein descriptor calculation, (3) pre-processing and feature selection,
model training, visualisation and validation, and (4) bioactivity/property prediction for new molecules. In the first instance, compound structures are
subjected to a common representation with the function StandardiseMolecules. Proteins are encoded with 8 types of amino acid and/or 13 types of
full protein sequence descriptors, whereas camb enables the calculation of 905 1D physicochemical descriptors for small molecules, and 14 types of
fingerprints, such as Morgan or Klekota fingerprints. Molecular descriptors are statistically pre-processed, e.g., by centering their values to zero mean
and scaling them to unit variance. Subsequently, single or ensemble machine learning models can be trained, visualised and validated. Finally, the
camb function PredictExternal allows the user (1) to read an external set of molecules with a trained model, (2) to apply the same processing to
these new molecules, and (3) to output predictions for this external set. This ensures that the same standardization options and descriptor types are
used when a model is applied to make predictions for new molecules.

Currently available $\mathrm{R}$ packages provide the capability for only subsets of the above mentioned steps. For instance, the $\mathrm{R}$ packages chemmineR [9] and $r c d k$ [10] enable the manipulation of SDF and SMILES files, the calculation of physicochemical descriptors, the clustering of molecules, and the retrieval of compounds from PubChem [3]. On the machine learning side, the caret package provides a unified platform for the training of machine learning models [11].

While it is possible to use a combination of these packages to set up a desired workflow, going from start to finish requires a reasonable understanding of model building in caret.

Here, we present the $\mathrm{R}$ package camb: Chemically $A$ ware Model Builder, which aims to address the current lack of an $\mathrm{R}$ framework comprising the four steps mentioned above. Specifically, the camb package makes it extremely easy to enter new molecules (that have no previous standardisation) through a single function, to acquire new predictions once model building has been done. The package has been conceived such that users with minimal programming skills can generate competitive predictive models and high-quality plots showing the performance of the models under default operation. It must be noted that camb does limit practitioners to a limited but easily used workflow to begin with. Experienced users, or those that intend to practice machine learning in
$\mathrm{R}$ extensively are encouraged to neglect this basic wrapper completely on their second training attempt and learn how to use the caret package from the caret related vignettes directly.

Overall, $c a m b$ enables the generation of predictive models, such as Quantitative Structure-Activity Relationships (QSAR), Quantitative Structure-Property Relationships (QSPR), Quantitative Sequence-Activity Modelling (QSAM), or Proteochemometric Modelling (PCM), starting with: chemical structure files, protein sequences (if required), and the associated properties or bioactivities. Moreover, $c a m b$ is the first $\mathrm{R}$ package that enables the manipulation of chemical structures utilising Indigo's C API [12], and the calculation of: (1) molecular fingerprints and 1-D [13] topological descriptors calculated using the PaDEL-Descriptor Java library [14], (2) hashed and unhashed Morgan fingerprints [15], and (3) eight types of amino acid descriptors. Two case studies illustrating the application of camb for QSPR modelling (solubility prediction) and PCM are available in the Additional files $1,2$.

\section{Design and implementation}

This section describes the tools provided by camb for (1) compound standardisation, (2) descriptor calculation, (3) pre-processing and feature selection, model training, visualisation and validation, and (4) bioactivity/property prediction for new molecules. 


\section{Compound standardization}

Chemical structure representations are highly ambiguous if SMILES are used for representation-for example, when one considers aromaticity of ring systems, protonation states, and tautomers present in a particular environment. Hence, standardisation is a step of crucial importance when either storing structures or before descriptor calculation. Many molecular properties are dependent on a consistent assignment of the above criteria in the first place. If one examines large chemical databases one can see how important this step is-a rather good explanation for standardisation is found in PubChem, one of the largest public databases, can be found on the PubChem Blog [16]. Hence, we are of the opinion that standardising chemical structures is crucial in order to provide consistent data for later modelling steps, in line with perceptions by others (such as the PubChem curators). For standardisation, camb provides the function StandardiseMolecules which utilises Indigo's C API [12]. SDF and SMILES formats are provided as molecule input options. Any molecules that Indigo fails to parse are removed during the standardisation step. As a filter, the user can stipulate the maximum number of each halogen atom that a compound can possess in order to pass the standardisation process. This allows datasets with a bias towards many molecules that contain one type of halogen to be easily normalised before training. Additional arguments of this function include the removal of inorganic molecules or those compounds with a molecular mass above or below a defined threshold. Most importantly, camb makes use of Indigo's InChI [17] plugin to represent all tautomers by the same canonical SMILES by converting molecules to InChI, discarding tautomeric information, and converting back to SMILES.

\section{Descriptor calculation}

Currently, camb supports the calculation of compound descriptors and fingerprints via PaDEL-Descriptor [14], and Morgan circular fingerprints [15] as implemented in RDkit [18]. The function GeneratePadelDescriptors permits the calculation of 9051 - and 2-D descriptors and 10 PaDEL-Descriptor fingerprints, namely: CDK fingerprints [19], CDK extended fingerprints [19], KierHall E-state fragments [20], CDK graph only fingerprints [19], MACCS fingerprints [21], Pubchem fingerprints [3], Substructure fingerprints [22], and Klekota-Roth fingerprints [23].

In addition to the PaDEL-Descriptor fingerprints, Morgan fingerprints can be computed with the function MorganFPs through the python library RDkit [18]. Hashed fingerprints can be generated as binary, recording the presence or absence of each substructure, or count based, recording the number of occurrences of each substructure. Additionally, the MorganFPs function also computes unhashed (keyed) fingerprints, where each substructure in the dataset is assigned a unique position in a binary fingerprint of length equal to the number of substructures existing in the dataset. Since the positions of substructures in the unhashed fingerprint depend on the dataset, the function MorganFPs allows calculation of unhashed fingerprints for new compounds using a basis defined by the substructures present in the training dataset. This ensures that substructures in new compounds map to the same locations on the fingerprint and allows enhanced model interpretation by noting which exact substructures are deemed important by the learning algorithm.

The function SeqDescs enables the calculation of 13 types of whole protein sequence descriptors from UniProt identifiers or from amino acid sequences [24], namely: amino acid composition (AAC), dipeptide composition (DC), tripeptide composition (TC), normalized Moreau-Broto autocorrelation (MoreauBroto), Moran autocorrelation (Moran), Geary autocorrelation (Geary), CTD (composition/transition/distribution) (CTD), Conjoint Traid (CTriad), sequence order coupling number $(\mathrm{SOCN})$, quasi-sequence order descriptors (QSO), pseudo amino acid composition (PACC), amphiphilic pseudo amino acid composition (APAAC) $[25,26]$.

In addition, camb permits the calculation of 8 types of amino acid descriptors, namely: 3 and $5 \mathrm{Z}$-scales (Z3 and Z5), T-Scales (TScales), ST-Scales (STScales), Principal Components Score Vectors of Hydrophobic, Steric, and Electronic properties (VHSE), BLOSUM62 Substitution Matrix (BLOSUM), FASGAI (FASGAI), MSWHIM (MSWHIM), and ProtFP PCA8 (ProtFP8). Amino acid descriptors can be used for modelling of the activity of small peptides or for the description of protein binding sites $[5,25,27,28]$. Multiple sequence alignment gaps are supported by this camb functionality. Descriptor values for these gaps are encoded with zeros. Further details about these descriptors and their predictive signal for bioactivity modelling can be found in two recent publications $[25,26]$.

\section{Model training and validation}

Prior to model training, descriptors often need to be preprocessed [29] so that they are equally weighted as inputs into the learning algorithms and to remove any that contain little relevant information content. To this end, several functions (see package documentation and tutorials) are provided. These functions include the removal of non-informative descriptors (function RemoveNearZeroVarianceFeatures) or highly correlated descriptors (function RemoveHighlyCorrelatedFeatures), the imputation of missing descriptor values (function ImputeFeatures), and 
descriptor centering and scaling to unit variance (function PreProcess) among others [30].

The $\mathrm{R}$ package caret provides a common interface to the most popular machine learning packages that exist in R, and, as such, camb invokes caret to set up crossvalidation frameworks and train machine learning models. These include learning methods in Bagging, Bayesian Methods, Boosting, Boosted Trees, Elastic Net, MARS, Gaussian Processes, K Nearest Neighbour, Principal Component Regression, Radial Basis Function Networks, Random Forests, Relevance Vector Machines, and Support Vector Machines among others. Additionally, two ensemble modelling approaches, namely greedy and stacking optimisation, have been integrated from the $\mathrm{R}$ package caretEnsemble [31], which allows the combination of models to form ensemble models, which have proven to be less error prone [28].

In greedy optimization [32], the cross-validated RMSE is optimized using a linear combination of input model predictions. The input models are all trained using an identical fold composition. Each model is assigned a weight in the following manner. Initially, all models have their weight set to zero. The weight for a given model is repeatedly incremented by 1 if the subsequent normalized weight vector results in a closer match between the weighted combination of cross-validated predictions and the observed values (i.e. lower RMSE of the linear combination). This repetition is carried out $n$ times, by default $n=1,000$. The resulting weight vector is then normalized to obtain a final weight vector.

In the case of model stacking [28], the predictions of the input models serve as training data points for a meta-model. This meta-model can have linear, e.g. Partial Least Squares [33], or non-linear, e.g. Random Forest [34] characteristics. If the selected algorithm allows the importance of its inputs to be determined, each input corresponds to a single model, then the relative contributions of each model to the prediction can be ascertained. These model ensembles can be applied to a test set (which was not used when building the ensembles), and the error metric (e.g. RMSE) compared to that of the single models on the test set.

In the general case, prior to model training, the dataset is divided into a training set, comprising e.g. $70 \%$ of the data, and a test set, which comprises the remaining data. The test set is used to assess the predictive power of the models on new data points not considered in the training phase. In the training phase, the values of the model parameters (hyper-parameters) are optimized by grid search and $k$-fold cross-validation (CV) [35]. A grid of plausible hyper-parameter values covering an exponential range is defined (function expGrid). Next, the training set is split into $k$ folds by, e.g. stratified or random sampling of the bioactivity/property values. For each combination of hyper-parameters, a model is trained on $k-1$ folds, and the values for the remaining fold are then predicted. This procedure is repeated $k$ times, each time holding out a different fold. The values of the hyper-parameters exhibiting the lowest average RMSE (or another metric such as e.g. $R^{2}$ ) value across the $k$ folds are considered optimal. A model is then trained on the whole training set using the optimal hyper-parameter values, and the predictive power of this model is assessed on the test set. The final model, trained on the whole dataset after having optimized the hyper-parameter values by $\mathrm{CV}$, can be used to make predictions on an external chemical library.

Statistical metrics for model validation have also been included:

During cross-validation

$$
\begin{aligned}
& q_{C V}^{2} \text { or } R_{C V}^{2}=1-\frac{\sum_{i=1}^{N_{t r}}\left(y_{i}-\tilde{y}_{i}\right)^{2}}{\sum_{i=1}^{N_{t r}}\left(y_{i}-\bar{y}_{t r}\right)^{2}} \\
& \operatorname{RMSE}_{C V}=\sqrt{\frac{\left(y_{i}-\tilde{y}_{i}\right)^{2}}{N}}
\end{aligned}
$$

where $N_{t r}, y_{i}, \tilde{y}_{i}$ and $\overline{y_{t r}}$ represent the size of the training set, observation $i$, prediction $i$, and the average value of observations in the training set, respectively.

During testing

$$
\begin{aligned}
& Q_{1}^{2} \text { test }=1-\frac{\sum_{j=1}^{N_{\text {test }}}\left(y_{j}-\tilde{y}_{j}\right)^{2}}{\sum_{j=1}^{N_{\text {test }}}\left(y_{j}-\overline{y_{\text {tr }}}\right)^{2}} \\
& Q_{2}^{2} \text { test }=1-\frac{\sum_{j=1}^{N_{\text {test }}}\left(y_{j}-\tilde{y}_{j}\right)^{2}}{\sum_{j=1}^{N_{\text {test }}}\left(y_{j}-y_{\text {test }}\right)^{2}} \\
& Q_{3 \text { test }}^{2}=1-\frac{\left[\sum_{j=1}^{N_{\text {test }}}\left(y_{j}-\tilde{y}_{j}\right)^{2}\right] / N_{\text {test }}}{\left[\sum_{j=1}^{N_{t r}}\left(y_{j}-\overline{y_{t r}}\right)^{2}\right] / N_{t r}} \\
& R M S E_{\text {test }}=\sqrt{\frac{\left(y_{j}-\tilde{y}_{j}\right)^{2}}{N}} \\
& R_{\text {test }}=\frac{\sum_{j=1}^{N_{\text {test }}}\left(y_{j}-\bar{y}_{\text {test }}\right)\left(\tilde{y}_{j}-\tilde{y}_{\text {test }}{ }^{-}\right)}{\sqrt{\sum_{j=1}^{N_{\text {test }}}\left(y_{j}-\bar{y}_{\text {test }}\right)^{2} \sum\left(\tilde{y}_{j}-\tilde{y}_{\text {test }}{ }^{-}\right)^{2}}} \\
& R_{0 \text { test }}^{2}=1-\frac{\sum_{j=1}^{N_{\text {test }}}\left(y_{j}-\tilde{y}_{j}^{r 0}\right)^{2}}{\sum_{j=1}^{N_{\text {test }}}\left(y_{j}-y_{\text {test }}^{-}\right)^{2}}
\end{aligned}
$$


where $N_{t r}, N_{\text {test }}, y_{j}, \tilde{y}_{j}$, and $y_{\text {test }}^{-}$represent the size of the training and test sets, observation $j$, prediction $j$, and the average value of observations in the test set, respectively. $\bar{y}_{t r}$ represents the average value of observations in the training set.

$R_{0}^{2}$ test is the square of the coefficient of determination through the origin, being $\widetilde{y}_{j}^{r 0}=k \widetilde{y}_{j}$ the regression through the origin (observed versus predicted) and $k$ its slope. The reader is referred to Ref. [36] for a detailed discussion of both the evaluation of model predictive ability through the test set and about the three different formulations for $Q_{\text {test }}^{2}$, namely $Q_{1 \text { test }}^{2}, Q_{2}^{2}$ test , and $Q_{3}^{2}$ test. The value of these metrics permits the assessment of model performance according to the criteria proposed by Tropsha and Golbraikh [37, 38], namely: $q_{C V}^{2}>0.5, R_{\text {test }}^{2}>0.6$, $\frac{\left(R_{\text {test }}^{2}-R_{0}^{2} \text { test }\right.}{R_{\text {test }}^{2}}<0.1$, and $0.85 \leq k \leq 1.15$.

These values might change depending on the dataset modelled, as well as on the application context, e.g. higher errors might be tolerated in hit identification in comparison to lead optimization, nevertheless, these criteria can serve as general guidelines to assess model predictive ability. The function Validation permits the calculation of all these metrics.

In cases where information about the experimental error of the data is available, the values for the statistical metrics on the test set can be compared to the theoretical maximum and minimum achievable performance given (1) the uncertainty of the experimental measurements, (2) the size of the training and test sets, and (3) the distribution of the dependent variable [39]. The distribution of maximum and minimum $R_{0}^{2}$ test $, R_{\text {test }}, Q_{\text {test }}^{2}$, and $\mathrm{RMSE}_{\text {test }}$ values can be computed with the functions MaxPerf and MinPerf. The distributions of maximum model performance are calculated in the following way. A sample, $S$, of size equal to the test set is randomly drawn from the dependent variable, e.g. $\mathrm{IC}_{50}$ values. Next, the experimental uncertainty is added to $S$, which defines the sample $S_{\text {noise. }}$. The $R_{0 \text { test }}^{2}, R_{\text {test }}, Q_{\text {test }}^{2}$, and $\mathrm{RMSE}_{\text {test }}$ values for $S$ against $S_{\text {noise }}$ are then calculated. These steps are repeated $n$ times, by default 1,000 , to calculate the distributions of $R_{0 \text { test }}^{2}, R_{\text {test }}, Q_{\text {test }}^{2}$, and RMSE $E_{\text {test }}$ values. To calculate the distributions of minimum model performance, the same steps are followed, with the exception that $S$ is randomly permuted before calculating the values for the statistical metrics.

\section{Visualization}

Visualization functionality for model performance and for exploratory analyses of the data is provided. All plots are generated using the R package ggplot2 [40]. Default options of the plotting functions were chosen to allow the generation of high-quality plots, and in addition, the layer-based structure of ggplot objects allows for further optimisation by the addition of customisation layers. The visualization tools include correlation plots (CorrelationPlot), bar plots with error bars (ErrorBarplot), and Principal Component Analysis (PCA) (PCA and PCAPlot), histograms (DensityResponse), and pairwise distance distribution plots (PairwiseDistPlot). For instance, the camb function $P C A$ performs a Principal Component Analysis (PCA) on compound and/or protein descriptors. The output can be directly sent to the function PCAPlot, which will depict the two fist principal components, with the shape and color of a user-defined class e.g. compound class or protein isoform (Fig. 2).

Visual depiction of compounds is also possible with the function PlotMolecules, utilising Indigo's C API. Visualization functions are exemplified in the tutorials provided in the Additional file 2 and with the package documentation (folder $c a m b / d o c$ of the package).

\section{Predictions for new molecules}

One of the major benefits of having all tools available in one framework is that it is straightforward to perform exactly the same processing on new molecules as the

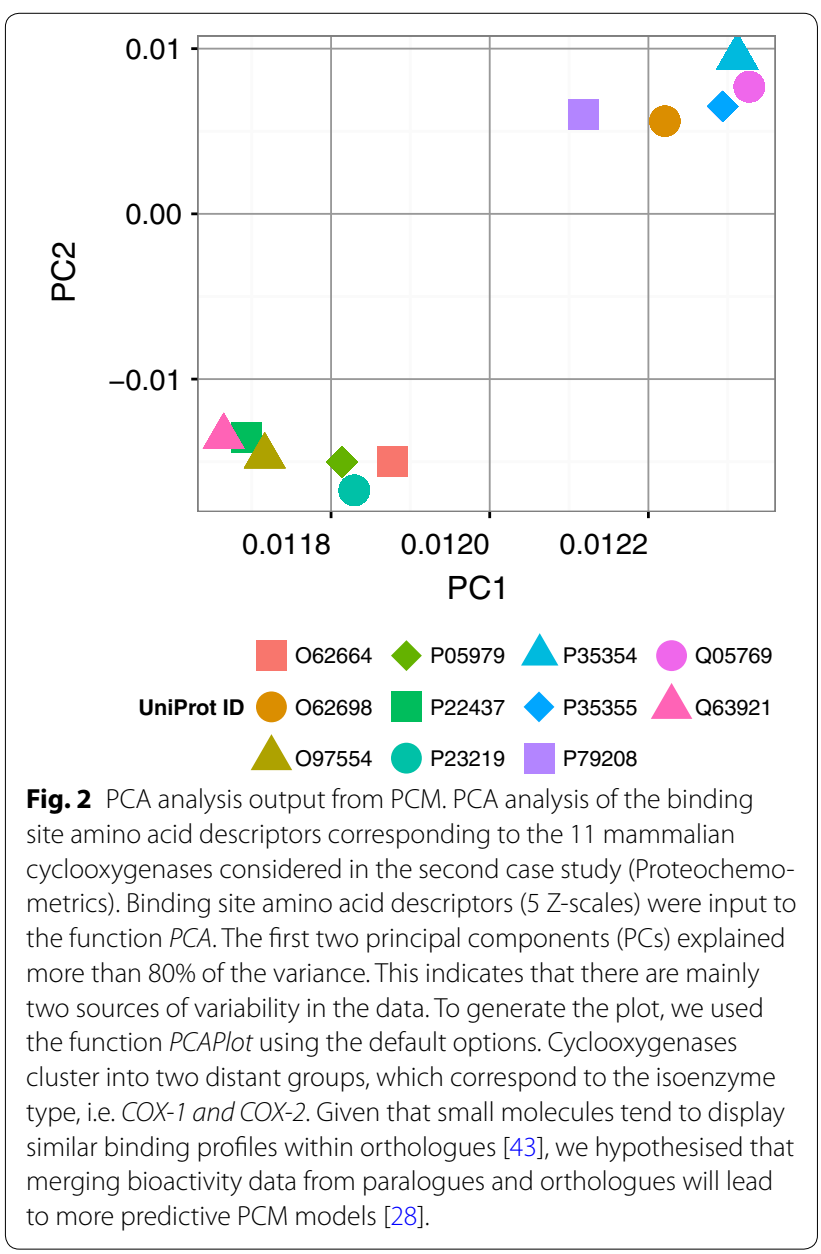


ones used on the training set, e.g. standardisation of molecules and centering and scaling of descriptors. The camb function PredictExternal allows the user to read an external set of molecules together with a trained model, and outputs predictions on this external set. This camb functionality ensures that the same standardization options and descriptor types are used when a model is applied to make predictions for new molecules. An example of this is shown in the QSPR tutorial.

\section{Results and discussion}

Two tutorials demonstrating property and bioactivity modelling are available as Additional files 1 and 2, and also within the package documentation. We encourage camb users to visit the package repository (https:// github.com/cambDI/camb) for future updated versions of the tutorials. In the following subsections, we show the results obtained for the two case studies presented in the tutorials, namely: (1) QSPR: prediction of compound aqueous solubility $(\operatorname{logS})$, and (2) PCM: modelling of the inhibition of 11 mammalian cyclooxygenases (COX) by small molecules. The datasets are available in the examples/PCM directory of the package. Further details about the PCM dataset can be found in Ref. [28].

\section{Case study 1: QSPR}

To illustrate the functionalities of camb for compound property modelling, the aqueous solubility values for 1,708 small molecules were downloaded [41]. Aqueous solubility values were expressed as $\log S$, where $\mathrm{S}$ corresponds to the solubility at a temperature of $20-25 \circ \mathrm{C}$ in $\mathrm{mol} / \mathrm{L}$. A common representation for the compound structures was found using the function StandardiseMolecules with default parameters, meaning that all molecules were kept irrespective of their molecular mass or the number of halogens present within their structure. Molecules were represented with implicit hydrogens, dearomatized, and passed through the InChI format to ensure that tautomers were represented by the same SMILES. 905 one and two-dimensional topological and physicochemical descriptors were then calculated using the function GeneratePadelDescriptors provided by the PaDEL-Descriptor [14] Java library built into the camb package. Missing descriptor values were imputed with the function ImputeFeatures. Two filtering steps were then performed: (1) highly-correlated descriptors with redundant predictive signal were removed using the function RemoveHighlyCorrelatedFeatures with a cut-off value of 0.95 , and (2) descriptors with near zero variance and hence limited predictive signal, were removed using the function RemoveNearZeroVarianceFeatures with a cut-off value of 30/1. Prior to model training, all descriptors were centered to have zero mean and scaled to have unit variance using the function PreProcess. After applying these steps the dataset consisted of 1,606 molecules encoded with 211 descriptors.

Three machine learning models were trained using $80 \%$ of the data (training set), namely: (1) Support Vector Machine (SVM) with a radial kernel, (2) Random Forest (RF), and (3) Gradient Boosting Machines (GBM). Fivefold cross-validation was used to optimize the value of the hyperparameters. Cross-validation and testing metrics for these three models are summarized in Table 1 . Overall, the three algorithms displayed high performance on the test set, with RMSE/ $R_{0}^{2}$ values of: GBM: 0.52/0.93; RF: 0.59/0.91; and SVM: 0.60/0.91 (Table 1; Fig. 3a). The combination of these three models as an ensemble was evaluated for improved predictive ability. To this end, two ensemble modelling techniques supported by camb were explored, namely: greedy optimization and model stacking. First, greedy ensemble was trained using the function caretEnsemble with 1,000 iterations. The greedy ensemble picked a linear combination of model outputs that was a local minimum in the RMSE landscape.

Table 1 Cross-validation and testing metrics for the single and ensemble QSPR models trained on the compound solubility dataset

\begin{tabular}{|c|c|c|c|c|}
\hline Algorithm & $R_{C V}^{2}$ & $\operatorname{RMSE}_{\mathrm{CV}}$ & $R_{0}^{2}$ test & RMSE $_{\text {test }}$ \\
\hline \multicolumn{5}{|l|}{ A } \\
\hline GBM & 0.90 & 0.59 & 0.93 & 0.52 \\
\hline $\mathrm{RF}$ & 0.89 & 0.62 & 0.91 & 0.59 \\
\hline SVM radial & 0.88 & 0.63 & 0.91 & 0.60 \\
\hline \multicolumn{5}{|l|}{ B } \\
\hline Greedy & - & 0.57 & 0.93 & 0.51 \\
\hline Linear stacking & 0.90 & 0.57 & 0.93 & 0.51 \\
\hline RF stacking & 0.89 & 0.62 & 0.92 & 0.55 \\
\hline
\end{tabular}

The lowest RMSE value on the test set, namely 0.51 , was obtained with the greedy and with the linear stacking ensembles.

GBM Gradient Boosting Machine, RF Random Forest, RMSE root mean square error, SVM Support Vector Machine. 

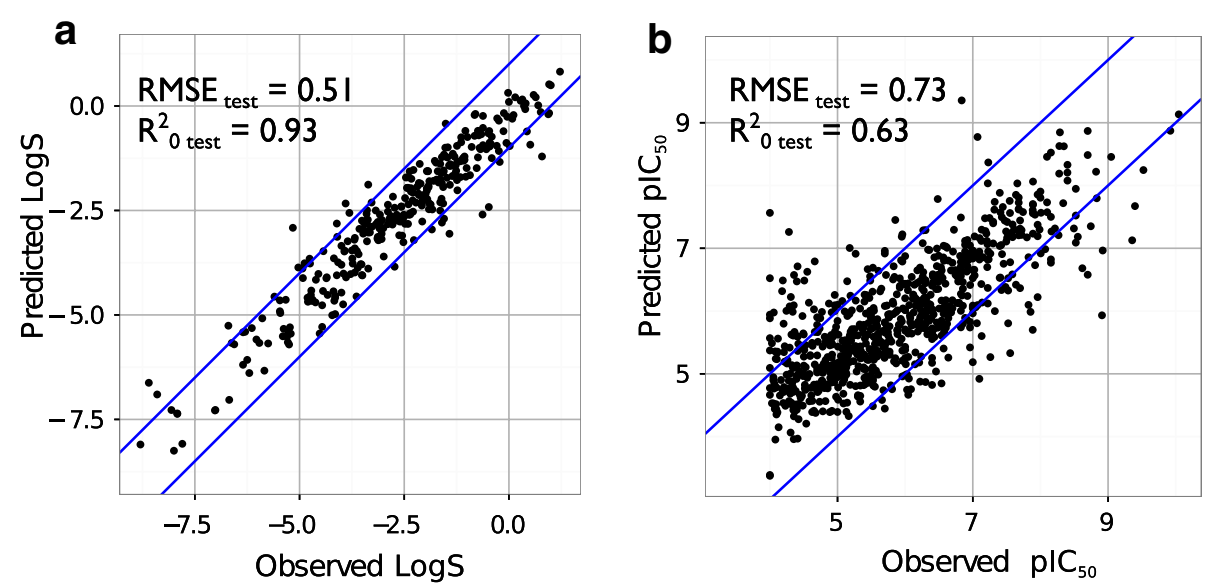

Fig. 3 Observed vs predicted for both case studies. Observed against predicted values on the test set corresponding to a the compound solubility (LogS) dataset (case study 1: QSPR), and $\mathbf{b}$ the cyclooxygenase (COX) inhibition dataset (case study 2: PCM). Both a and $\mathbf{b}$ were generated with the function CorrelationPlot. The area defined by the blue lines comprises 1 LogS units $(\mathbf{a})$ and 1 plC ${ }_{50}$ units (b). Both plots were generated using the predictions on the test set calculated with the Linear Stacking ensembles (Tables 1, 3). Overall, high predictive power is attained on the test set for both datasets, with respective RMSE/R2 values of 0.51/0.93 (a), and 0.73/0.63 (b). Taken together, these data indicate that ensemble modelling leads to higher predictive power, although this increase might be marginal for some datasets (b).

Secondly, linear and non-linear stacking ensembles were created. In model stacking, the cross-validated predictions of a library of models are used as descriptors, on which a meta-model (ensemble model) is trained. This meta model can be a linear model, e.g. SVM with a linear kernel, or non linear, such as Random Forest. The application of ensemble modelling led to a decrease by $10-15 \%$ of $\mathrm{RMSE}_{\text {test }}$ values (Table 1). The highest predictive power was obtained with the greedy and the linear stacking ensembles, with $R_{0}^{2}$ test $/ \mathrm{RMSE}_{\text {test }}$ of $0.93 / 0.51$ and $0.93 / 0.51$, respectively. Taken together, these results indicate that higher predictive power can be obtained when modelling this dataset by combining different single QSPR models with either greedy optimisation or model stacking. From this case study it can be seen that by utilizing the $c a m b$ package, a model training task which might involve porting datasets between multiple different external tools can be simplified to a few lines of code in a reproducible fashion within the $\mathrm{R}$ language alone. Additionally, predictions can easily be made on new molecules using a single function call passing in a new structures file.

\section{Case study 2: proteochemometrics}

In the second case study the functionalities of $c a m b$ are illustrated for proteochemoemtric modelling. The tutorial "PCM with camb" (Additional file 2) reports the complete modelling pipeline for this dataset [28]. Bioactivity data for 11 mammalian COX (COX-1 and COX-2 inhibitors) was extracted from ChEMBL 16 [2, 28] (Table 2). Only the data satisfying the following criteria
Table 2 Cyclooxygenase inhibition dataset ("Results and discussion" section, case study 2 )

\begin{tabular}{llll}
\hline UniProt ID & Isoenzyme & Organism & $\begin{array}{l}\text { Number of } \\
\text { datapoints }\end{array}$ \\
\hline P23219 & 1 & Homo sapiens & 1,346 \\
O62664 & 1 & Box taurus & 48 \\
P22437 & 1 & Mus musculus & 50 \\
O97554 & 1 & Oryctolagus cuniculus & 11 \\
P05979 & 1 & Ovis aries & 442 \\
Q63921 & 1 & Rattus norvegicus & 23 \\
P35354 & 2 & Homo sapiens & 2,311 \\
O62698 & 2 & Bos taurus & 21 \\
Q05769 & 2 & Mus musculus & 305 \\
P79208 & 2 & Ovis aries & 341 \\
P35355 & 2 & Rattus norvegicus & 39 \\
\hline
\end{tabular}

We extracted the bioactivity data for 11 mammalian cyclooxygenases from ChEMBL 16 [2]. The final bioactivity selection comprised 3,228 distinct compounds.

was kept: (1) assay score confidence higher than 8, (2) activity relationship equal to '=,', (3) activity type equal to "IC50", and (4) activity unit equal to 'nM'. The mean $\mathrm{IC}_{50}$ value was taken for duplicated compound-COX combinations. The final dataset comprised 3,228 distinct compounds and 11 mammalian COX proteins, with a total number of 4,937 datapoints (13.9\% matrix completeness) [28].

A common representation for the compound structures was found using the function StandardiseMolecules with default parameters. Then, two main 
descriptor types were calculated: (1) PaDEL descriptors [14] with the function GeneratePadelDescriptors, (2) and Morgan fingerprints with the function MorganFPs. Substructures with a maximal diameter of 4 bonds were considered. The length of the fingerprints was set to 512. To describe the target space, the binding site amino acid descriptors were derived from the crystallographic structure of ovine COX-1 complexed with celecoxib (PDB ID: 3KK6 [42]) by selecting those residues within a sphere of radius equal to 10 $\AA$ centered in the ligand. Subsequently, we performed multiple sequence alignment to determine the corresponding residues for the other $10 \mathrm{COX}$, and calculated $5 \mathrm{Z}$-scales for these residues with the function AADescs.

Prior to model training, missing descriptor values were imputed (function ImputeFeatures). Two filtering steps were then performed: (1) highly-correlated descriptors with redundant predictive signal were removed using the function RemoveHighlyCorrelatedFeatures with a cut-off value of 0.95 , and (2) descriptors with near zero variance and hence limited predictive signal, were removed using the function RemoveNearZeroVarianceFeatures with a cut-off value of 30/1. Prior to model training, all descriptors were centered to have zero mean and scaled to have unit variance using the function PreProcess. These steps led to a final selection of 356 descriptors: 242 Morgan fingerprint binary descriptors, 99 physicochemical descriptors, and $15 Z$-scales. The dataset was split into a training set, which was comprised of $80 \%$ of the data, and a test set (20\%) with the function SplitSet. Three single PCM models were trained using fivefold cross-validation, namely: GBM, RF, and SVM with a radial kernel (Table 3).
These models were subsequently combined into model ensembles using (1) greedy optimisation (1,000 iterations), and (2) model stacking (Table 3). The function Validation served to calculate the values for the statistical metrics on the test set. The observed against the predicted values on the test set were reported with the function CorrelationPlot (Fig. 3b).

All model ensembles displayed higher predictive power on the test set than single PCM models, except for RF Stacking (Table 3). The lowest RMSE value on the test set, namely 0.72 was obtained with the Elastic Network (EN) Stacking model (Table 3), whereas the highest $R_{0}^{2}$ value, namely 0.63 , was obtained with the greedy, the Linear Stacking and the SVM Radial Stacking ensembles. As in the previous case study, these data indicate that higher predictive power can be obtained by combining single PCM models in more predictive model ensembles, although this improvement might be sometimes marginal. This case study illustrates the versatility of camb to train and validate PCM models from amino acid sequences and compound structures in an integrated and seamless modelling pipeline.

\section{Availability and future directions}

camb is coded in $\mathrm{R}, \mathrm{C}++$, Python and Java and is available open source at https://github.com/cambDI/camb. To install camb from R type: library(devtools); install_ github("cambDI/camb/camb"). We plan to include further functionality based on the $\mathrm{C}++$ Indigo API, and to implement new error estimation methods for regression and classification models. Additionally, we plan to further integrate the python library RDkit with camb. The package is fully documented and includes the usage examples and details of the $\mathrm{R}$ functions implemented in camb.

Table 3 Cross-validation and testing metrics for the single and ensemble PCM models trained on the COX dataset

\begin{tabular}{|c|c|c|c|c|}
\hline Algorithm & $R_{C V}^{2}$ & $\mathrm{RMSE}_{\mathrm{CV}}$ & $R_{0}^{2}$ test & RMSE $_{\text {test }}$ \\
\hline \multicolumn{5}{|l|}{ A } \\
\hline GBM & 0.59 & 0.77 & 0.60 & 0.76 \\
\hline $\mathrm{RF}$ & 0.60 & 0.78 & 0.61 & 0.79 \\
\hline SVM & 0.61 & 0.75 & 0.60 & 0.76 \\
\hline \multicolumn{5}{|l|}{ B } \\
\hline Greedy ensemble & - & 0.73 & 0.63 & 0.73 \\
\hline Linear stacking & 0.63 & 0.73 & 0.63 & 0.73 \\
\hline EN stacking & 0.63 & 0.72 & 0.62 & 0.72 \\
\hline SVM linear stacking & 0.63 & 0.73 & 0.62 & 0.73 \\
\hline SVM radial stacking & 0.63 & 0.73 & 0.63 & 0.73 \\
\hline RF stacking & 0.61 & 0.76 & 0.58 & 0.77 \\
\hline
\end{tabular}

Combining single models trained with different algorithms in model ensembles allows to increase model predictive ability. We obtained the highest $R_{0}^{2}$ test and $\mathrm{RMSE}_{\text {test }}$ values namely, 0.63 and $0.73 \mathrm{plC}_{50}$ unit respectively, with the greedy ensemble, and with the following model stacking techniques: (1) linear, and (2) SVM radial.

EN Elastic Net, GBM Gradient Boosting Machine, RF Random Forest, RMSE root mean square error in prediction, SVM Support Vector Machines. 


\section{Conclusions}

In silico predictive models have proved valuable for the optimisation of compound potency, selectivity and safety profiles. In this context, camb provides an open framework to (1) compound standardisation, (2) molecular and protein descriptor calculation, (3) pre-processing and feature selection, model training, visualisation and validation, and (4) bioactivity/property prediction for new molecules. All the above functionalities will speed up model generation, provide reproducibility and tests of robustness. camb functions have been designed to meet the needs of both expert and amateur users. Therefore, $c a m b$ can serve as an education platform for undergraduate, graduate, and post-doctoral students, while providing versatile functionalities for predictive bioactivity/property modelling in more advanced settings.

\section{Additional files}

Additional file 1: QSPR with camb.pdf-Tutorial demonstrating the utility of the camb [1] package by presenting a pipeline which generates various aqueous solubility models using2D molecular descriptors calculated by the PaDEL-Descriptor package as input features. These models are then ensembled to create asingle model with a greater predictive accuracy. The trained ensemble is then put to use in making predictions for new molecules. Additional file 2: PCM with camb.pdf-Tutorial demonstrating a pipeline to generate a Proteochemometric (PCM) model for mammal cyclooxygenase (COX) inhibitors. Further details about this dataset are reserved for a future publication. Similarly, the interested reader is referred to ref [1] and [2] forfurther details about PCM.

\section{Authors' contributions}

DM and ICC conceived and coded the package. DM and ICC wrote the tutorials. GvW provided analytical tools for amino acid descriptor calculation. DM, ICC, GVW, IS, AB, TM and RG wrote the paper. All authors read and approved the final manuscript.

\section{Author details}

${ }^{1}$ Department of Chemistry, Centre for Molecular Informatics, University of Cambridge, Lensfield Road, Cambridge CB2 1EW, UK. ${ }^{2}$ Unite de Bioinformatique Structurale, Structural Biology and Chemistry Department, Institut Pasteur and CNRS UMR 3825, 25-28, rue Dr. Roux, 75724 Paris, France.

${ }^{3}$ European Molecular Biology Laboratory, European Bioinformatics Institute, Wellcome Trust Genome Campus, Hinxton CB101SD, UK. ${ }^{4}$ Unilever Research, Port Sunlight Laboratory, Bebington L63 3JW, Wirral, UK.

\section{Acknowledgements}

ICC thanks the Paris-Pasteur International PhD Programme and Institut Pasteur for funding. TM thanks CNRS and Institut Pasteur for funding. DSM and RCG thanks Unilever for funding. GvW thanks EMBL (EIPOD) and Marie Curie (COFUND) for funding. AB thanks Unilever and the European Research Commission (Starting Grant ERC-2013-StG 336159 MIXTURE) for funding.

\section{Compliance with ethical guidelines}

\section{Competing interests}

The authors declare that they have no competing interests.

Received: 1 April 2015 Accepted: 3 July 2015

Published online: 28 August 2015

\section{References}

1. Bender A (2010) Databases: compound bioactivities go public. Nat Chem Biol 6(5):309

2. Gaulton A, Bellis LJ, Bento AP, Chambers J, Davies M, Hersey A et al (2011) ChEMBL: a large-scale bioactivity database for drug discovery. Nucleic Acids Res. 40(D1):1100-1107

3. Wang Y, Xiao J, Suzek TO, Zhang J, Wang J, Zhou Z et al (2012) PubChem's BioAssay Database. Nucleic Acids Res 40(Database issue):400-412

4. van Westen GJP, Wegner JK, IJzerman AP, van Vlijmen HWT, Bender A (2011) Proteochemometric modeling as a tool to design selective compounds and for extrapolating to novel targets. Med Chem Comm 2:16-30

5. Cortes Ciriano I, Ain QU, Subramanian V, Lenselink EB, Mendez Lucio O, IJzerman AP et al (2015) Polypharmacology modelling using proteochemometrics: recent developments and future prospects. Med Chem Comm 6:24-50

6. R Core Team (2013) R: a language and environment for statistical computing. R Foundation for Statistical Computing, Vienna, Austria. http:// www.R-project.org

7. Gentleman RC, Carey VJ, Bates DM, Bolstad B, Dettling M, Dudoit S et al (2004) Bioconductor: open software development for computational biology and bioinformatics. Genome Biol 5:80

8. Mente S, Kuhn M (2012) The use of the R language for medicinal chemistry applications. Curr Top Med Chem 12(18):1957-1964

9. Cao Y, Charisi A, Cheng LC, Jiang T, Girke T (2008) ChemmineR: a compound mining framework for R. Bioinformatics 24(15):1733-1734

10. Guha R (2007) Chemical informatics functionality in R. J Stat Softw 18(5):1-16

11. Kuhn M (2008) Building predictive models in r using the caret package. J Stat Softw 28(5):1-26

12. Indigo (2013) Indigo Cheminformatics Library. GGA Software Services, Cambridge

13. Rognan D (2007) Chemogenomic approaches to rational drug design. $\mathrm{Br}$ J Pharmacol 152(1):38-52

14. Yap CW (2011) PaDEL-Descriptor: an open source software to calculate molecular descriptors and fingerprints (v2.16). J Comput Chem 32(7):1466-1474

15. Rogers D, Hahn M (2010) Extended-connectivity fingerprints. J Chem Inf Model 50(5):742-754

16. PubChem (2014) PubChem Blog: what is the difference between a substance and a compound in PubChem?. http://pubchemblog.ncbi.nlm. nih.gov/2014/06/19/what-is-the-difference-between-a-substance-and-acompound-in-pubchem/

17. InChl (2013) IUPAC - International Union of Pure and Applied Chemistry: The IUPAC International Chemical Identifier (InChl). http://www.iupac. org/home/publications/e-resources/inchi.html

18. Landrum G (2006) RDKit: open-source cheminformatics. http://www. rdkit.org

19. Steinbeck C, Han Y, Kuhn S, Horlacher O, Luttmann E, Willighagen E (2003) The chemistry development kit (cdk): an open-source java library for chemo- and bioinformatics. J Chem Inf Comput Sci 43(2):493-500

20. Hall LH, Kier LB (1995) Electrotopological state indices for atom types: a novel combination of electronic, topological, and valence state information. J Chem Inf Comput Sci 35(6):1039-1045

21. Durant JL, Leland BA, Henry DR, Nourse JG (2002) Reoptimization of mdl keys for use in drug discovery. J Chem Inf Comput Sci 42(6):1273-1280

22. O'Boyle NM, Banck M, James CA, Morley C, Vandermeersch T, Hutchison GR (2011) Open Babel: an open chemical toolbox. J Cheminf 3(1):33

23. Klekota J, Roth FP (2008) Chemical substructures that enrich for biological activity. Bioinformatics 24(21):2518-2525

24. Xiao N, Xu Q (2014) Protr: Protein sequence descriptor calculation and similarity computation with R. R package version 0.2-1

25. van Westen GJ, Swier RF, Cortes-Ciriano I, Wegner JK, Overington JP, Ijzerman AP et al (2013) Benchmarking of protein descriptor sets in proteochemometric modeling (part 2): Modeling performance of 13 amino acid descriptor sets. J Cheminf 5(1):42

26. van Westen G, Swier R, Wegner JK, IJzerman AP, van Vlijmen HW, Bender A (2013) Benchmarking of protein descriptor sets in proteochemometric modeling (part 1): comparative study of 13 amino acid descriptor sets. J Cheminf 5(1):41 
27. van Westen GJP, van den Hoven OO, van der Pijl R, Mulder-Krieger T, de Vries H, Wegner JK et al (2012) Identifying novel adenosine receptor ligands by simultaneous proteochemometric modeling of rat and human bioactivity data. J Med Chem 55(16):7010-7020

28. Cortes-Ciriano I, Murrell DS, van Westen GJP, Bender A, Malliavin T (2014) Prediction of the potency of mammalian cyclooxygenase inhibitors with ensemble proteochemometric modeling. J Cheminf 7:1

29. Andersson CR, Gustafsson MG, Strömbergsson H (2011) Quantitative chemogenomics: machine-learning models of protein-ligand interaction. Curr Top Med Chem 11(15):1978-1993

30. Kuhn M, Johnson K (2013) Applied predictive modeling. Springer, New York

31. Mayer Z (2013) CaretEnsemble: framework for combining caret models into ensembles. [R Package Version 1.0]

32. Caruana R, Niculescu-Mizil A, Crew G, Ksikes A (2004) Ensemble selection from libraries of models. In: Proceedings of the 21 st international conference on machine learning. ICML'04, ACM, New York, p 18

33. Wold S, Sjöström M, Eriksson L (2001) PLS-regression: a basic tool of chemometrics. Chemometr Intell Lab 58(2):109-130

34. Breiman $L$ (2001) Random forests. Mach Learn 45(1):5-32

35. Hawkins DM, Basak SC, Mills D (2003) Assessing model fit by cross-validation. J Chem Inf Comput Sci 43(2):579-586

36. Consonni V, Ballabio D, Todeschini R (2010) Evaluation of model predictive ability by external validation techniques. J. Chemom 24(3-4):194-201

37. Golbraikh A, Tropsha A (2002) Beware of q2!. J Mol Graphics Modell 20(4):269-276
38. Tropsha A, Gramatica P, Gombar VK (2003) The importance of being earnest: validation is the absolute essential for successful application and interpretation of QSPR models. QSAR Comb Sci 22(1):69-77

39. Cortes Ciriano I, van Westen GJ, Lenselink EB, Murrell DS, Bender A, Malliavin T (2014) Proteochemometric modeling in a Bayesian framework. J Cheminf 6(1):35

40. Wickham H (2009) Ggplot2: elegant graphics for data analysis. http://had. co.nz/ggplot2/book

41. Wang J, Krudy G, Hou T, Zhang W, Holland G, Xu X (2007) Development of reliable aqueous solubility models and their application in druglike analysis. J Chem Inf Model 47(4):1395-1404

42. Rimon G, Sidhu RS, Lauver DA, Lee JY, Sharma NP, Yuan C, Frieler RA, Trievel RC, Lucchesi BR, Smith WL (2010) Coxibs interfere with the action of aspirin by binding tightly to one monomer of cyclooxygenase-1. Proc Natl Acad Sci USA 107(1):28-33

43. Kruger FA, Overington JP (2012) Global analysis of small molecule binding to related protein targets. PLoS Comput Biol 8(1):1002333

\section{Publish with ChemistryCentral and every scientist can read your work free of charge \\ "Open access provides opportunities to our colleagues in other parts of the globe, by allowing anyone to view the content free of charge."}

W. Jeffery Hurst, The Hershey Company.

- available free of charge to the entire scientific community

- peer reviewed and published immediately upon acceptance

- cited in PubMed and archived on PubMed Central

- yours - you keep the copyright

Submit your manuscript here:

http://www.chemistrycentral.com/manuscript/ 